\title{
Intensive glucose control and macrovascular outcomes in type 2 diabetes
}

\author{
F. M. Turnbull • C. Abraira • R. J. Anderson - R. P. Byington - J. P. Chalmers - \\ W. C. Duckworth - G. W. Evans - H. C. Gerstein - R. R. Holman - T. E. Moritz • \\ B. C. Neal • T. Ninomiya • A. A. Patel $\cdot$ S. K. Paul F. Travert $\cdot$ M. Woodward
}

Received: 18 June 2009 / Accepted: 19 June 2009 / Published online: 5 August 2009

(C) Springer-Verlag 2009

\begin{abstract}
Aims/hypothesis Improved glucose control in type 2 diabetes is known to reduce the risk of microvascular events. There is, however, continuing uncertainty about its impact on macrovascular disease. The aim of these analyses was to generate more precise estimates of the effects of more-intensive, compared with less-intensive, glucose control on the risk of major cardiovascular events amongst patients with type 2 diabetes.

Methods A prospectively planned group-level metaanalysis in which characteristics of trials to be included,
\end{abstract}

Received: 18 June 2009 / Accepted on the basis of reviews submitted from another journal: 19 June 2009.

Authors are members of the Collaborators on Trials of Lowering

Glucose (CONTROL) writing group.

Electronic supplementary material The online version of this article (doi:10.1007/s00125-009-1470-0) contains supplementary material, which is available to authorised users.

F. M. Turnbull $(\bowtie) \cdot$ J. P. Chalmers $\cdot$ B. C. Neal $\cdot$ T. Ninomiya $\cdot$ A. A. Patel $\cdot$ M. Woodward

The George Institute for International Health, University of Sydney,

PO Box M201, Missenden Rd,

Sydney, NSW 2050, Australia

e-mail: fturnbull@george.org.au

C. Abraira

Miami Veterans Affairs Medical Center,

Miami, FL, USA

\section{R. J. Anderson - T. E. Moritz}

Hines Veterans Affairs Cooperative Studies

Program Coordinating Center,

Hines, IL, USA

R. P. Byington · G. W. Evans

Wake Forest University School of Medicine,

Winston-Salem, NC, USA outcomes of interest, analyses and subgroup definitions were all pre-specified.

Results A total of 27,049 participants and 2,370 major vascular events contributed to the meta-analyses. Allocation to more-intensive, compared with less-intensive, glucose control reduced the risk of major cardiovascular events by $9 \%$ (HR $0.91,95 \%$ CI $0.84-0.99$ ), primarily because of a $15 \%$ reduced risk of myocardial infarction (HR 0.85 , 95\% CI 0.76 0.94). Mortality was not decreased, with non-significant HRs of 1.04 for all-cause mortality (95\% CI $0.90-1.20)$ and 1.10 for cardiovascular death (95\% CI $0.84-1.42)$. Intensively

W. C. Duckworth

Phoenix Veterans Affairs Health Care Center,

Phoenix, AZ, USA

H. C. Gerstein

McMaster University and Hamilton Health Sciences,

Population Health Research Institute,

Hamilton, ON, Canada

R. R. Holman · S. K. Paul

Oxford Centre for Diabetes, Endocrinology, and Metabolism,

Oxford, UK

F. Travert

Centre d'Investigations Cliniques, Groupe Hospitalier Bichat Claude Bernard, Assistance Publique des Hôpitaux de Paris, Paris, France 
treated participants had significantly more major hypoglycaemic events (HR 2.48, 95\% CI 1.91-3.21). Exploratory subgroup analyses suggested the possibility of a differential effect for major cardiovascular events in participants with and without macrovascular disease (HR 1.00, 95\% CI 0.89-1.13, vs HR 0.84 , 95\% CI $0.74-0.94$, respectively; interaction $p=0.04)$.

Conclusions/interpretation Targeting more-intensive glucose lowering modestly reduced major macrovascular events and increased major hypoglycaemia over 4.4 years in persons with type 2 diabetes. The analyses suggest that glucoselowering regimens should be tailored to the individual.

Keywords Meta-analysis · Randomised trials · Intensive glucose control - Macrovascular outcomes · Hypoglycaemia

$\begin{array}{ll}\begin{array}{l}\text { Abbreviations } \\ \text { ACCORD }\end{array} & \begin{array}{l}\text { Action to Control Cardiovascular Risk in } \\ \text { Diabetes }\end{array} \\ \text { ADVANCE } & \begin{array}{l}\text { Action in Diabetes and Vascular Disease: } \\ \text { Preterax and Diamicron Modified Release }\end{array} \\ & \begin{array}{l}\text { Controlled Evaluation } \\ \text { UKPDS }\end{array} \\ \text { UK Prospective Diabetes Study } \\ \text { Veterans Affairs Diabetes Trial }\end{array}$

\section{Introduction}

Diabetes is a serious chronic disease that is growing rapidly in prevalence and that now affects $>10 \%$ of adults in developed countries $[1,2]$. People with type 2 diabetes are two to four times more likely to develop a serious cardiovascular outcome compared with those without diabetes [3, 4]. Despite risk-reduction strategies that include lowering of cholesterol and BP, and smoking cessation, the majority of those with diabetes continue to die from cardiovascular causes [5]. The degree to which improved glucose control could help address this residual cardiovascular risk remains uncertain.

The UK Prospective Diabetes Study (UKPDS) showed that hyperglycaemia, as assessed by $\mathrm{HbA}_{1 \mathrm{c}}$ levels, was a statistically independent and potentially modifiable risk factor for cardiovascular disease, in addition to LDLcholesterol, HDL-cholesterol, BP and smoking [6]. Findings

\footnotetext{
M. Woodward

Mount Sinai Medical Center,

New York, NY, USA

R. J. Anderson

Division of Epidemiology and Biostatistics School of Public Health, University of Illinois,

Chicago, IL, USA
}

from other large observational studies have confirmed the continuous and positive association between various measures of glycaemia (including fasting and post-load glucose levels and $\mathrm{HbA}_{1 \mathrm{c}}$ ) and the risk of cardiovascular disease [7, 8]. However, despite achieving a median $0.9 \% \mathrm{HbA}_{1 \mathrm{c}}$ difference for a median of 10 years, the UKPDS did not demonstrate a statistically significant risk reduction for myocardial infarction.

The results of three other clinical trials [9-11] designed primarily to determine whether targeting lower vs higher glucose levels can reduce the risk of cardiovascular events in patients with type 2 diabetes were published in 2008. Of these, the ACCORD trial's intensive glycaemic intervention [9] was terminated early after a median of 3.5 years because of higher mortality among participants assigned to an $\mathrm{HbA}_{1 \mathrm{c}}$ target of $<6.0 \%$.

In order to provide more precise estimates of the effects of glucose-lowering on major cardiovascular events, the Action to Control Cardiovascular Risk in Diabetes (ACCORD) [9], Action in Diabetes and Vascular Disease: Preterax and Diamicron Modified Release Controlled Evaluation (ADVANCE) [10], UKPDS [12] and Veterans Affairs Diabetes Trial (VADT) [11] investigators have established a collaboration to facilitate a formal metaanalysis of the results from each trial and to explore any differences among trials.

\section{Methods}

Trial inclusion and exclusion criteria Trials were eligible for inclusion in these meta-analyses if they were designed to assess directly the impact of achieving lower vs higher levels of glycaemia on cardiovascular outcomes in adult patients with type 2 diabetes and had the following features: large size, defined as at least 1,000 person-years of follow-up in each treatment arm and a minimum of 2 years median postrandomisation follow-up; randomised and controlled; doubleblind or blind assessment of endpoint design; pre-specified cardiovascular outcomes; analysed using an intention-to-treat approach; and follow-up of $\geq 90 \%$ of randomised participants for vital status.

Trials randomising individuals to comprehensive cardiovascular risk-reduction strategies were excluded unless there was a separate randomisation to different levels of glycaemic control. Trials conducted in patients with type 1 diabetes, with gestational diabetes or in children aged $\leq 16$ years and trials conducted in acute or critical-care settings were all excluded.

Search strategy A comprehensive literature search was performed to determine if any trials in addition to ACCORD, ADVANCE, UKPDS and VADT met the inclusion and 
exclusion criteria for this review. Potentially eligible trials were identified using literature searches, scrutiny of clinical trial registers and abstracts of proceedings, and enquiry among colleagues and industry representatives, in accordance with internationally accepted norms. MEDLINE, controlled clinical trial registers and the Cochrane Database were searched for articles published in English up until January 2009. Studies were identified through PubMed searches of the MEDLINE database with the MeSH headings 'blood glucose', 'diabetes mellitus', 'clinical trial', and the non-MeSH terms 'glycaemic/glycemic/blood glucose control' and 'aggressive/tight/intensive'. Reference lists of the retrieved articles were also searched to identify other eligible studies, and information from colleagues was used to identify morerecently published articles.

Outcomes All outcomes were pre-specified in the study protocol for this meta-analysis. The primary outcome was a composite of major cardiovascular events, defined as death from cardiovascular causes (including sudden death), nonfatal myocardial infarction and non-fatal stroke.

Secondary outcomes were stroke (non-fatal and fatal), myocardial infarction (non-fatal and fatal), heart failure resulting in hospitalisation or death, cardiovascular death, non-cardiovascular death, all-cause mortality and major hypoglycaemia. Major hypoglycaemia was defined as an episode with typical symptoms and signs of hypoglycaemia, without other apparent cause, where the individual was unable to treat $\mathrm{him} / \mathrm{herself}$.

Data collection Each trial group collated data from its own trial according to pre-specified variable definitions and shared group data with each of the other three groups. This enabled collaborators to cross-check the data and to perform the analyses independently. UKPDS data were censored at 5 years after randomisation, so as to provide a follow-up duration that was similar to that of the other three trials. Individual patient data meta-analysis was not done, as ongoing within-trial analyses are in progress and one trial (ACCORD) [9] is continuing to follow participants within the active-treatment phase of the study.

Statistical analysis The overall mean age of participants, duration of follow-up and proportion of women was calculated using the mean values for each trial weighted by the trial's number of participants. The glycaemic separation achieved between randomised groups for mean $\mathrm{HbA}_{1 \mathrm{c}}$ and for mean fasting plasma glucose in each trial was calculated as the differences in the reductions between baseline and the last recorded visit. The overall glycaemic separation between randomised groups was calculated as the inverse-variance weighted mean of the individual trial differences using a random-effects model.
HRs for the impact on outcomes of intensive vs lessintensive glycaemic control were estimated separately for each trial using Cox proportional hazards models and according to intention-to-treat. Overall effect estimates, and $95 \%$ CIs, were calculated using the random-effects model. (i.e. weighting by the statistical precision of the estimate in each trial). Sensitivity analyses were performed using fixed-effects models. The cut-off for significance with respect to the primary outcome of macrovascular disease was set at $p<0.05(95 \%$ CIs exclude 1.0$)$; the same cut-off was identified for the secondary outcomes, in the event of a significant primary outcome. All tests were two-sided. There were no adjustments made for multiple comparisons. Heterogeneity across studies was estimated using the $I^{2}$ statistic, which measures the percentage of variability across studies that is attributable to heterogeneity rather than chance, and was tested using the $Q$ test with a cut-off for significance of $p=0.1$ chosen before the analyses began [13]. For each trial, HRs and 95\% CIs were also calculated cumulatively for each year of follow-up, to examine the effect of length of follow-up on the primary cardiovascular outcome.

Pre-defined subgroup analyses were performed to explore the effect of therapy on major cardiovascular events. Common definitions for the subgroups were used across all the trials and the subgroups assessed included sex, age, duration of known diabetes, pre-existing macrovascular disease, preexisting microvascular disease and baseline $\mathrm{HbA}_{1 \mathrm{c}}$. Consistency of treatment effects across the subgroups was tested using $\chi^{2}$ tests of homogeneity. Because no adjustments for multiple comparisons were made for secondary outcomes or for subgroups, these analyses should be regarded as exploratory.

Analyses were carried out using STATA (Release 9.2; Stata Corporation, College Station, TX, USA).

\section{Results}

Of 163 studies that were identified from the literature search, only four (ACCORD [9], ADVANCE [10], UKPDS [12] and VADT [11]) satisfied the study eligibility criteria for the meta-analysis (Electronic supplementary material [ESM] Fig. 1). Key characteristics of these four trials are shown in Table 1. Between them they randomised a total of 27,049 participants, the majority of whom were selected on the basis of having type 2 diabetes in conjunction with at least one other risk factor for cardiovascular disease. The ACCORD, ADVANCE and VADT trials allocated participants equally to intensive vs less-intensive glycaemic treatment groups; the UKPDS allocated $70 \%$ of its participants to an intensive group and $30 \%$ to the less-intensive group. Median 
Fig. 1 Effects of more- vs lessintensive glycaemic control on major cardiovascular events (cardiovascular death or non-fatal stroke or non-fatal myocardial infarction), stroke (fatal or non-fatal), myocardial infarction (fatal or non-fatal) and heart failure resulting in hospitalisation or death. The diamond incorporates the point estimate, represented by the vertical dashed line, and the $95 \% \mathrm{CI}$ of the overall effect for each outcome. The HRs are given for more-intensive compared with less-intensive glucose control. $\Delta \mathrm{HbA}_{1 \mathrm{c}}=$ mean $\mathrm{HbA}_{1 \mathrm{c}}$ of more-intensive group minus mean $\mathrm{HbA}_{1 \mathrm{c}}$ of less-intensive group. UKPDS follow-up truncated at 5 years from the time of randomisation

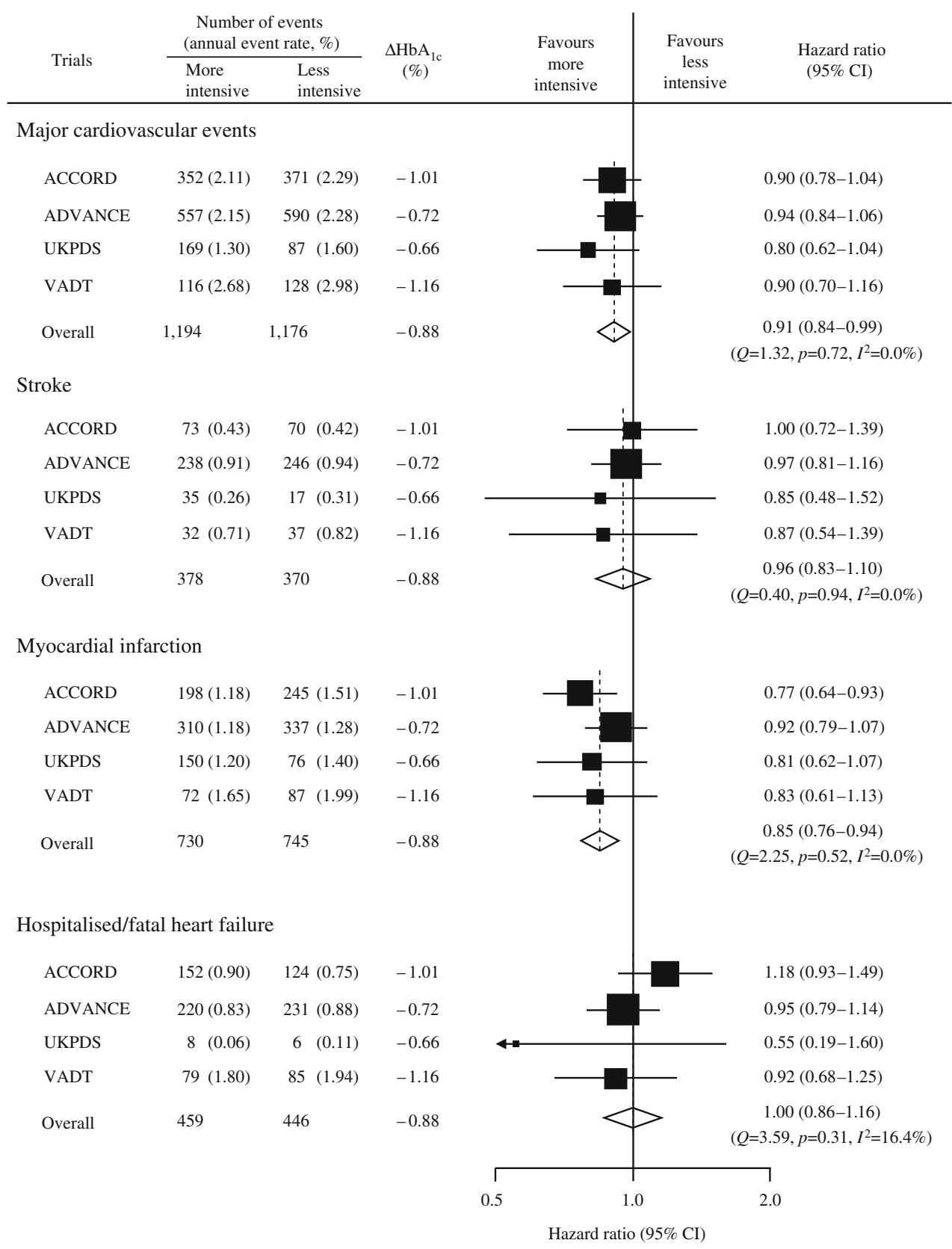

follow-up visit, participants allocated to more-intensive glycaemic control were taking more glucose-lowering therapies but the proportions taking other risk-factor treatments did not differ between randomised groups, although there were differences among the trials (Table 3 ).

Table 4 shows the mean differences achieved in glycaemic control and the mean differences observed in other major risk factors between randomised groups at the last clinic visit. The overall weighted mean $\mathrm{HbA}_{1 \mathrm{c}}$ and fasting plasma glucose differences between those allocated to more- compared with less-intensive glycaemic control were 0.88 percentage points (Table 5) and $1.53 \mathrm{mmol} / \mathrm{l}$ (data not shown), respectively. 
Table 1 Key characteristics of trials and length of follow-up

\begin{tabular}{|c|c|c|c|c|c|c|c|}
\hline Trial name & $\begin{array}{l}\text { Trial } \\
\text { acronym }\end{array}$ & $\begin{array}{l}\text { Year } \\
\text { reported }\end{array}$ & Number & Design & $\begin{array}{l}\text { Glycaemic control } \\
\text { comparison }\end{array}$ & Entry criteria & $\begin{array}{l}\text { Median } \\
\text { follow-up } \\
\text { (years) }\end{array}$ \\
\hline $\begin{array}{l}\text { The Action to Control } \\
\text { Cardiovascular Risk in } \\
\text { Diabetes Study }\end{array}$ & ACCORD & 2008 & 10,251 & $\begin{array}{l}\text { Randomised, } \\
\text { double } 2 \times 2 \\
\text { factorial }\end{array}$ & $\begin{array}{l}\text { Intensive } \\
\quad \text { (target } \mathrm{HbA}_{1 \mathrm{c}}<6 \% \text { ) } \\
\left.\text { vs standard (target } \mathrm{HbA}_{1 \mathrm{c}} 7-7.9 \%\right)\end{array}$ & $\begin{array}{l}\text { Type } 2 \text { diabetes, } \\
\mathrm{HbA}_{1 \mathrm{c}} \geq 7.5 \%, \\
40-79 \text { years or } \\
55-79 \text { years }^{\mathrm{a}}\end{array}$ & $3.4^{\mathrm{b}}$ \\
\hline $\begin{array}{l}\text { Action in Diabetes and } \\
\text { Vascular Disease: } \\
\text { Preterax }{ }^{\mathrm{g}}+\text { Diamicron } \\
\text { Modified Release } \\
\text { Controlled Evaluation }\end{array}$ & ADVANCE & 2008 & 11,140 & $\begin{array}{l}\text { Randomised, } \\
2 \times 2 \text { factorial }\end{array}$ & $\begin{array}{l}\text { Intensive (target } \\
\left.\mathrm{HbA}_{1 \mathrm{c}} \leq 6.5 \%\right) \text { vs } \\
\text { standard (target } \\
\left.\mathrm{HbA}_{1 \mathrm{c}}>6.5 \%\right)\end{array}$ & $\begin{array}{l}\text { Diagnosis of type } \\
2 \text { diabetes at } \\
\geq 30 \text { years, } \\
\geq 55 \text { years }\end{array}$ & 4.9 \\
\hline $\begin{array}{l}\text { UK Prospective } \\
\text { Diabetes Study }\end{array}$ & UKPDS & 1998 & 3,867 & Randomised & $\begin{array}{l}\text { Intensive (target FPG } \\
<6 \mathrm{mmol} / \mathrm{l} \text { ) vs } \\
\text { conventional } \\
\text { (best achievable FPG } \\
\text { with diet alone) }\end{array}$ & $\begin{array}{l}\text { Newly diagnosed } \\
\text { type } 2 \text { diabetes, } \\
25-65 \text { years old }^{\mathrm{d}}\end{array}$ & $5.0^{\mathrm{e}}$ \\
\hline $\begin{array}{l}\text { Veterans Affairs } \\
\text { Diabetes Trial }\end{array}$ & VADT & 2008 & 1,791 & Randomised & $\begin{array}{l}\text { Intensive (target } \\
\text { absolute reduction } \\
1.5 \% \text { ) vs standard }\end{array}$ & $\begin{array}{l}\text { Poorly controlled } \\
\text { type } 2 \text { diabetes, } \\
\text { military veterans }{ }^{\mathrm{f}}\end{array}$ & 5.6 \\
\hline
\end{tabular}

The Recommended International Non-proprietary Name (rINN) for Diamicron is gliclazide

a 40-79 years old and cardiovascular disease, 55-79 years old and significant atherosclerosis, albuminuria, left ventricular hypertrophy or at least two additional risk factors for cardiovascular disease

${ }^{\mathrm{b}}$ Discontinuation of intensive therapy after mean of 3.5 year follow-up because of higher mortality in intensively treated group

${ }^{\mathrm{c}}$ With history of major macrovascular disease or microvascular disease or at least one other risk factor for vascular disease

${ }^{\mathrm{d}}$ Fasting plasma glucose $>6 \mathrm{mmol} / \mathrm{l}$ on two mornings $1-3$ weeks apart following diagnosis by primary-care practitioner and $>6.0 \mathrm{but} \leq 15.0 \mathrm{mmol} / \mathrm{l}$ after 3-4 month dietary run-in

${ }^{\mathrm{e}}$ Follow-up truncated at 5.0 years for the purposes of this meta-analysis

${ }^{\mathrm{f}}$ Inadequate response to maximal doses of an oral agent or insulin therapy

${ }^{\mathrm{g}}$ Perindopril and indapamide fixed combination

FPG, fasting plasma glucose

Clinical outcomes A total of 2,370 major cardiovascular events $(1,194 / 14,320$ in the more-intensive group, 1,176/ 12,729 in the less-intensive group) contributed to the metaanalyses. The risk of a major cardiovascular event was reduced by $9 \%$ (Fig. 1) in those allocated to more-intensive compared with less-intensive glycaemic control (HR 0.91, 95\% CI 0.84-0.99) with no evidence of heterogeneity among trials $(p=0.72)$. The cumulative HRs by year of follow-up (ESM Table 1) did not differ appreciably and showed no systematic trend.

The risk of non-fatal/fatal myocardial infarction was reduced by $15 \%$ (Fig. 1) in those allocated to moreintensive compared with less-intensive glycaemic control (HR $0.85,95 \%$ CI $0.76-0.94$ ), with a non-significant reduction in the risk of non-fatal/fatal stroke (HR 0.96, 95\% CI $0.83-1.10$ ) and no difference for hospitalised fatal heart failure (HR 1.00, 95\% CI 0.86-1.16).

A total of 1,864 participants died. The HR for all-cause mortality among randomised groups was $1.04(95 \%$ CI $0.90-1.20$ ) (Fig. 2). Although the $Q$ test for heterogeneity did not reach statistical significance $(p=0.13)$, almost
$50 \%$ of variability across studies was estimated to be attributable to heterogeneity rather than chance $\left(I^{2}=47.5 \%\right)$. Cause of death could be categorised as cardiovascular or non-cardiovascular in all but $18(0.1 \%)$ participants. The estimated HR for cardiovascular death varied among trials ( $p$ for heterogeneity $=0.04$ ) but the overall estimate did not differ significantly from unity (HR $1.10,95 \%$ CI 0.84 1.42). Re-analysis using the fixed-effect model did not alter the results (ESM Table 2).

Effects on severe hypoglycaemia Overall, there were 1,443 events $(1,071$ in the more-intensive group, 372 in the lessintensive group). Allocation to more-intensive glycaemic control was associated with a more than doubling in the risk of severe hypoglycaemia (HR 2.48, 95\% CI 1.91-3.21) and there was significant heterogeneity among the trials $\left(Q=10.74, p=0.01, I^{2}=72.1 \%\right)$ (Table 5).

Subgroup analyses The effect of more-intensive glycaemic control on major cardiovascular events was consistent across pre-specified participant subgroups (Fig. 3) with 
Table 2 Participant characteristics at baseline

\begin{tabular}{|c|c|c|c|c|}
\hline Characteristic & $\begin{array}{l}\text { ACCORD } \\
(n=10,251)\end{array}$ & $\begin{array}{l}\text { ADVANCE } \\
(n=11,140)\end{array}$ & $\begin{array}{l}\text { UKPDS } \\
(n=3,867)\end{array}$ & $\begin{array}{l}\text { VADT } \\
(n=1,791)\end{array}$ \\
\hline \multicolumn{5}{|l|}{ Demographic characteristics } \\
\hline Age (years), mean (SD) & $62.2(6.8)$ & $65.8(6.4)$ & $53.3(8.6)$ & $60.4(8.7)$ \\
\hline Female, $n(\%)$ & $3,952(38.6)$ & $4,733(42.5)$ & $1,508(39.0)$ & $52(2.9)$ \\
\hline Age when diabetes first diagnosed (years), mean (SD) & NA & $57.8(8.7)$ & $53.3(8.6)$ & $48.9(10.0)$ \\
\hline Duration of known diabetes (years), median (Q1, Q3) & $10(5,15)$ & $7(3,11)$ & $0(0,0)$ & $10(6,16)$ \\
\hline \multicolumn{5}{|l|}{ Prior vascular disease } \\
\hline History of macrovascular disease, $n(\%)^{\mathrm{a}}$ & $3,608(35.2)$ & $3,590(32.2)$ & $77(2.0)$ & $723(40.4)$ \\
\hline History of microvascular disease, $n(\%)^{\mathrm{b}}$ & $1,778(17.4)$ & $1,155(10.4)$ & $73(1.9)$ & $185(14.10)^{\mathrm{c}}$ \\
\hline \multicolumn{5}{|l|}{ Glycaemic control } \\
\hline $\mathrm{HbA}_{1 \mathrm{c}}(\%)$, mean $(\mathrm{SD})$ & $8.3(1.1)$ & $7.5(1.6)$ & $7.1(1.5)^{\mathrm{d}}$ & $9.4(1.5)$ \\
\hline $\mathrm{HbA}_{1 \mathrm{c}}(\%)$, median $(\mathrm{Q} 1, \mathrm{Q} 3)$ & $8.1(7.6,8.9)$ & $7.2(6.5,8.2)$ & $6.8(5.9,7.9)^{\mathrm{d}}$ & $9.1(8.3,10.2)$ \\
\hline Fasting plasma glucose (mmol/1), mean (SD) & $9.7(3.1)$ & $8.5(2.8)$ & $8.4(2.3)^{\mathrm{d}}$ & $11.3(3.8)$ \\
\hline Fasting plasma glucose $(\mathrm{mmol} / \mathrm{l})$, median $(\mathrm{Q} 1, \mathrm{Q} 3)$ & $9.3(7.7,11.3)$ & $7.9(6.6,9.7)$ & $8.0(7.1,9.7)^{\mathrm{d}}$ & $10.7(8.5,13.6)$ \\
\hline \multicolumn{5}{|l|}{ Other major risk factors } \\
\hline Systolic BP (mmHg), mean (SD) & $136.4(17.1)$ & $145.0(21.5)$ & $135(20)$ & $131.6(16.7)$ \\
\hline Diastolic BP (mmHg), mean (SD) & $74.9(10.7)$ & $80.7(10.9)$ & $82(10)$ & $76.1(10.3)$ \\
\hline Total cholesterol (mmol/1), mean (SD) & $4.7(1.1)$ & $5.2(1.2)$ & $5.4(1.1)$ & $4.8(1.2)$ \\
\hline LDL-cholesterol (mmol/l), mean (SD) & $2.7(0.9)$ & $3.1(1.0)$ & $3.5(1.0)$ & $2.8(0.8)$ \\
\hline HDL-cholesterol (mmol/l), mean (SD) & $1.1(0.3)$ & $1.3(0.4)$ & $1.1(0.24)$ & $0.9(0.3)$ \\
\hline Triacylglycerols (mmol/1), median (Q1, Q3) & $1.8(1.2,2.6)$ & $1.6(1.2,2.3)$ & $2.4(0.8,6.5)$ & $1.8(1.3,2.7)$ \\
\hline Microalbuminuria, $n(\%)^{\mathrm{e}}$ & $2,501(24.6)$ & $2,857(26.9)^{\mathrm{f}}$ & $251(6.5)$ & $569(31.8)$ \\
\hline BMI $\left(\mathrm{kg} / \mathrm{m}^{2}\right)$, mean $(\mathrm{SD})$ & $32.2(5.5)$ & $28.3(5.2)$ & $27.5(5.2)$ & $31.2(4.4)$ \\
\hline Current smoking, $n(\%)$ & $1,429(14.0)$ & $1,550(13.9)$ & $1,199(31.0)$ & $299(16.7)$ \\
\hline \multicolumn{5}{|l|}{ Glucose-lowering treatment } \\
\hline Sulfonylurea, $n(\%)$ & $5,136(50.1)$ & 7,899 (70.9) & $0(0)$ & $1,090(60.9)$ \\
\hline Metformin, $n(\%)$ & $6,135(59.8)$ & $6,752(60.6)$ & $0(0)$ & $1,237(69.1)$ \\
\hline Thiazolidinedione, $n(\%)$ & $1,982(19.3)$ & $407(3.7)$ & $0(0)$ & $337(18.8)$ \\
\hline Acarbose $^{\mathrm{g}}, n(\%)$ & $69(0.7)$ & $960(8.6)$ & $0(0)$ & $36(2.0)$ \\
\hline Glinide, $n(\%)$ & $186(1.8)$ & $187(1.7)$ & $0(0)$ & $9(0.50)$ \\
\hline Insulin, $n(\%)$ & $3,581(34.9)$ & $159(1.4)$ & $0(0)$ & $938(52.4)$ \\
\hline
\end{tabular}

a Definition of 'History of macrovascular disease' varied slightly among studies. ACCORD: history of stroke, myocardial infarction, angina with ischaemic changes, coronary artery bypass graft or revascularisation; ADVANCE: history of stroke, transient ischaemic attack, myocardial infarction, angina, coronary artery bypass graft, revascularisation or amputation; UKPDS: history of myocardial infarction in the previous year, current angina or heart failure, or history of more than one major vascular event; VADT: history of stroke, transient ischaemic attack, myocardial infarction, angina, congestive heart failure, invasive revascularisation, amputation or intermittent claudication

${ }^{\mathrm{b}}$ Definition of 'History of microvascular disease' varied slightly among studies. ACCORD: history of urinary albumin/creatinine ratio (UACR) $>300 \mathrm{mg} / \mathrm{g}$ (to convert from $\mathrm{mg} / \mathrm{g}$ to $\mathrm{mg} / \mathrm{mmol}$, divide by 8.8401 ), retinopathy or blindness; ADVANCE: history of UACR $>300 \mathrm{mg} / \mathrm{g}$, proliferative retinopathy, macular oedema or diabetes-related blindness; UKPDS: retinopathy requiring photocoagulation; VADT: history of UACR $>300 \mathrm{mg} / \mathrm{g}$, proliferative retinopathy

${ }^{\mathrm{c}}$ Limited to 1,312 participants $(73 \%)$ who had fundus photographs at baseline

${ }^{\mathrm{d}}$ Measured after 3-4 months of dietary run-in

e $30 \leq \mathrm{UACR} \leq 300 \mathrm{mg} / \mathrm{g}$ except UKPDS ( $50 \leq$ urinary albumin concentration $<300 \mathrm{mg} / \mathrm{l}$ )

${ }^{\mathrm{f}}$ Baseline UACR data not available for 502 (4.5\%) of participants

${ }^{\mathrm{g}}$ Defined in ACCORD at baseline as any alpha glucosidase inhibitor use

$\mathrm{Q} 1$, first quartile; Q3, third quartile 
Table 3 Glucose-lowering and cardioprotective therapies at follow-up ${ }^{\mathrm{a}}$

\begin{tabular}{|c|c|c|c|c|c|c|c|c|}
\hline \multirow[t]{2}{*}{ Therapy } & \multicolumn{2}{|c|}{ ACCORD $(n=10,208)$} & \multicolumn{2}{|c|}{ ADVANCE $(n=10,973)$} & \multicolumn{2}{|c|}{$\mathrm{UKPDS}^{\mathrm{b}}(n=3,646)$} & \multicolumn{2}{|c|}{ VADT $(n=1,745)$} \\
\hline & $\begin{array}{l}\text { Less- } \\
\text { intensive }\end{array}$ & $\begin{array}{l}\text { More- } \\
\text { intensive }\end{array}$ & $\begin{array}{l}\text { Less- } \\
\text { intensive }\end{array}$ & $\begin{array}{l}\text { More- } \\
\text { intensive }\end{array}$ & $\begin{array}{l}\text { Less- } \\
\text { intensive }\end{array}$ & $\begin{array}{l}\text { More- } \\
\text { intensive }\end{array}$ & $\begin{array}{l}\text { Less- } \\
\text { intensive }\end{array}$ & $\begin{array}{l}\text { More- } \\
\text { intensive }\end{array}$ \\
\hline \multicolumn{9}{|l|}{ Glucose-lowering drugs } \\
\hline Sulfonylurea, $n(\%)$ & $2,516(49.3)$ & $2,304(45.1)$ & $3,245(59.1)$ & $4,939(90.1)$ & $273(25.6)$ & $1,384(53.7)$ & $387(44.1)$ & $461(53.1)$ \\
\hline Metformin, $n(\%)$ & $3,506(68.8)$ & $3,784(74.1)$ & $3,599(65.6)$ & $3,951(72.0)$ & $89(8.3)$ & $203(7.9)$ & $474(54.1)$ & $519(59.8)$ \\
\hline Thiazolidinedione, $n(\%)$ & $1,534(30.1)$ & $2,814(55.1)$ & $578(10.5)$ & $895(16.3)$ & N/A & N/A & $249(28.4)$ & $317(36.5)$ \\
\hline Acarbose, $n(\%)$ & $162(3.2)$ & $681(13.3)$ & $640(11.7)$ & $972(17.7)$ & N/A & N/A & $20(2.3)$ & $92(10.6)$ \\
\hline Glinide, $n(\%)$ & $487(9.6)$ & $1,280(25.0)$ & $145(2.6)$ & $70(1.3)$ & $\mathrm{N} / \mathrm{A}$ & $\mathrm{N} / \mathrm{A}$ & $2(0.23)$ & $10(1.2)$ \\
\hline Insulin, $n(\%)$ & $2,603(51.0)$ & $3,628(71.0)$ & $1,326(24.2)$ & $2,205(40.2)$ & $165(15.5)$ & $1,006(39.0)$ & $678(77.3)$ & $756(87.2)$ \\
\hline \multicolumn{9}{|l|}{ Other drugs ${ }^{\mathrm{c}}$} \\
\hline $\begin{array}{l}\text { Aspirin/other antiplatelet, } \\
n(\%)\end{array}$ & $2,953(59.4)$ & $2,912(58.8)$ & $3,216(58.6)$ & $3,302(60.2)$ & $51(4.8)$ & $104(4.0)$ & $662(75.7)$ & $658(75.9)$ \\
\hline $\begin{array}{l}\text { Statin/other lipid-lowering, } \\
n(\%)\end{array}$ & $3,924(79.0)$ & $3,797(76.7)$ & $2,801(51.1)$ & $2,739(49.9)$ & N/A & N/A & $590(67.5)$ & $609(70.2)$ \\
\hline $\begin{array}{l}\text { One or more BP-lowering drug, } \\
n(\%)\end{array}$ & $4,270(85.9)$ & $4,123(83.3)$ & $4,412(80.4)$ & $4,374(79.8)$ & $258(24.2)$ & $648(25.1)$ & $652(74.6)$ & $656(75.7)$ \\
\hline
\end{tabular}

a 'At follow-up' defined as the last available measure for each specified variable

${ }^{\mathrm{b}}$ Follow-up truncated at 5.0 years for the purposes of this meta-analysis

${ }^{\mathrm{c}}$ Restricted to 9,919 participants completing at least one annual follow-up examination

N/A, these agents were not available during the early part of the UKPDS

Table 4 Mean differences ${ }^{\mathrm{a}}$ in glycaemic levels and other risk factors at follow-up ${ }^{\mathrm{b}}$ between groups randomised to more- or less-intensive glucose control

\begin{tabular}{|c|c|c|c|c|}
\hline Variable & $\begin{array}{l}\text { ACCORD } \\
(n=10,208)\end{array}$ & $\begin{array}{l}\text { ADVANCE } \\
(n=10,977)\end{array}$ & $\begin{array}{l}\mathrm{UKPDS}^{\mathrm{c}} \\
(n=3,867)\end{array}$ & $\begin{array}{l}\text { VADT } \\
(n=1,745)\end{array}$ \\
\hline \multicolumn{5}{|l|}{ Glycaemic control } \\
\hline $\mathrm{HbA}_{1 \mathrm{c}}(\%)$, mean difference (SE) & $-1.01(0.03)$ & $-0.72(0.03)$ & $-0.66(0.08)$ & $-1.16(0.09)$ \\
\hline $\begin{array}{l}\text { Fasting plasma glucose }(\mathrm{mmol} / \mathrm{l}) \text {, } \\
\text { mean difference }(\mathrm{SE})\end{array}$ & $-2.01(0.07)$ & $-1.19(0.06)$ & $-1.40(0.14)$ & $-1.52(0.24)$ \\
\hline \multicolumn{5}{|l|}{ Other major risk factors } \\
\hline $\begin{array}{l}\text { BP, systolic/diastolic }(\mathrm{mmHg}) \\
\text { mean difference }(\mathrm{SE})\end{array}$ & $\begin{array}{l}-0.86(0.90) /-0.80 \\
(0.20)\end{array}$ & $\begin{array}{l}-2.26(0.42) /-1.18 \\
(0.22)\end{array}$ & $\begin{array}{l}-3.90(0.40) /-0.47 \\
(0.39)\end{array}$ & $\begin{array}{l}0.11(0.99) /-0.38 \\
(0.56)\end{array}$ \\
\hline Total cholesterol $(\mathrm{mmol} / \mathrm{l})$, mean difference $(\mathrm{SE})$ & $\mathrm{N} / \mathrm{A}$ & $-0.08(0.02)$ & $-0.05(0.04)$ & $-0.03(0.07)$ \\
\hline LDL-cholesterol (mmol/l) mean difference (SE) & $0.03(0.02)$ & $-0.02(0.02)$ & $-0.01(0.03)$ & \\
\hline HDL-cholesterol (mmol/l), mean difference (SE) & $\mathrm{N} / \mathrm{A}$ & $-0.01(0.01)$ & $-0.02(0.01)$ & $-0.02(0.01)$ \\
\hline $\begin{array}{l}\text { Triaclglycerols (log transformed, mmol/l), mean } \\
\text { difference (SE) }\end{array}$ & $\mathrm{N} / \mathrm{A}$ & $-0.06(0.01)$ & $-0.01(0.01)$ & $-0.04(0.03)$ \\
\hline Microalbuminuria, proportion difference, $\%$ (SE) & N/A & $-2.66(1.31)$ & $-4.66(1.34)$ & $-4.96(2.76)$ \\
\hline BMI $\left(\mathrm{kg} / \mathrm{m}^{2}\right)$, mean difference $(\mathrm{SE})$ & $0.97(0.06)$ & $0.35(0.05)$ & $0.79(0.04)$ & $1.45(0.16)$ \\
\hline Current smoking, difference in proportions (SE) & $-0.47(0.57)^{\mathrm{d}}$ & $-0.10(0.87)$ & $2.75(0.74)$ & $-0.28(1.43)$ \\
\hline
\end{tabular}

${ }^{a}$ Mean differences calculated as the reduction from baseline in the mean value (mean reduction intensive group-mean reduction standard group)

b 'At follow-up' defined as the last available measure for each specified variable

c Follow-up truncated at 5.0 years for the purposes of this meta-analysis

${ }^{\mathrm{d}}$ Restricted to 9,919 participants completing at least one annual follow-up examination

N/A, data not available for meta-analysis 
Table 5 Effects of more- vs less-intensive glycaemic control on severe hypoglycaemia

\begin{tabular}{|c|c|c|c|c|c|c|}
\hline \multirow[t]{2}{*}{ Trial } & \multicolumn{2}{|c|}{ More-intensive } & \multicolumn{2}{|c|}{ Less-intensive } & \multirow[t]{2}{*}{$\Delta \mathrm{HbA}_{1 \mathrm{c}}(\%)$} & \multirow[t]{2}{*}{ HR $(95 \%$ CI) } \\
\hline & No. at risk & No. of events & No. at risk & No of events & & \\
\hline ACCORD & 5,128 & 538 & 5,123 & 179 & -1.01 & $3.07(2.59-3.63)$ \\
\hline ADVANCE & 5,571 & 150 & 5,569 & 81 & -0.72 & $1.86(1.42-2.44)$ \\
\hline $\mathrm{UKPDS}^{\mathrm{a}}$ & 2,729 & 194 & 1,138 & 23 & -0.66 & $3.01(1.75-5.16)$ \\
\hline VADT & 892 & 189 & 899 & 89 & -1.16 & $2.30(1.79-2.96)$ \\
\hline Overall & 14,320 & 1,071 & 12,729 & 372 & -0.88 & $\begin{array}{l}2.48(1.91-3.21) \\
\left(Q=10.74[p=0.01], I^{2}=72.1 \%\right)\end{array}$ \\
\hline
\end{tabular}

${ }^{\text {a } U K P D S ~ e v e n t ~ n u m b e r s ~ m a y ~ b e ~ a n ~ u n d e r e s t i m a t e ~ a s ~ h y p o g l y c a e m i c ~ e p i s o d e s ~ w e r e ~ r e c o r d e d ~ a s ~ ' n o n e ' ~ o r ~ ' o n e ~ o r ~ m o r e ' ~ i n ~ e a c h ~} 3$ month followup period

one exception: participants who did not have a history of macrovascular disease prior to randomisation appeared to benefit from more-intensive glycaemic control, whereas those with a history of a macrovascular disease did not appear to benefit (test for homogeneity $p=0.04$ ). The UKPDS only enrolled participants with newly diagnosed diabetes and therefore this trial contributed data to one subgroup only for the analyses of 'duration of diabetes' and very few to the subgroups for 'history of macrovascular disease' and 'history of microvascular disease'. Sensitivity analyses excluding UKPDS data showed no material difference in the subgroup findings.
Fig. 2 Effects of more- vs less-intensive glycaemic control on all-cause mortality, cardiovascular death and non-cardiovascular death. Conventions as for Fig. 1

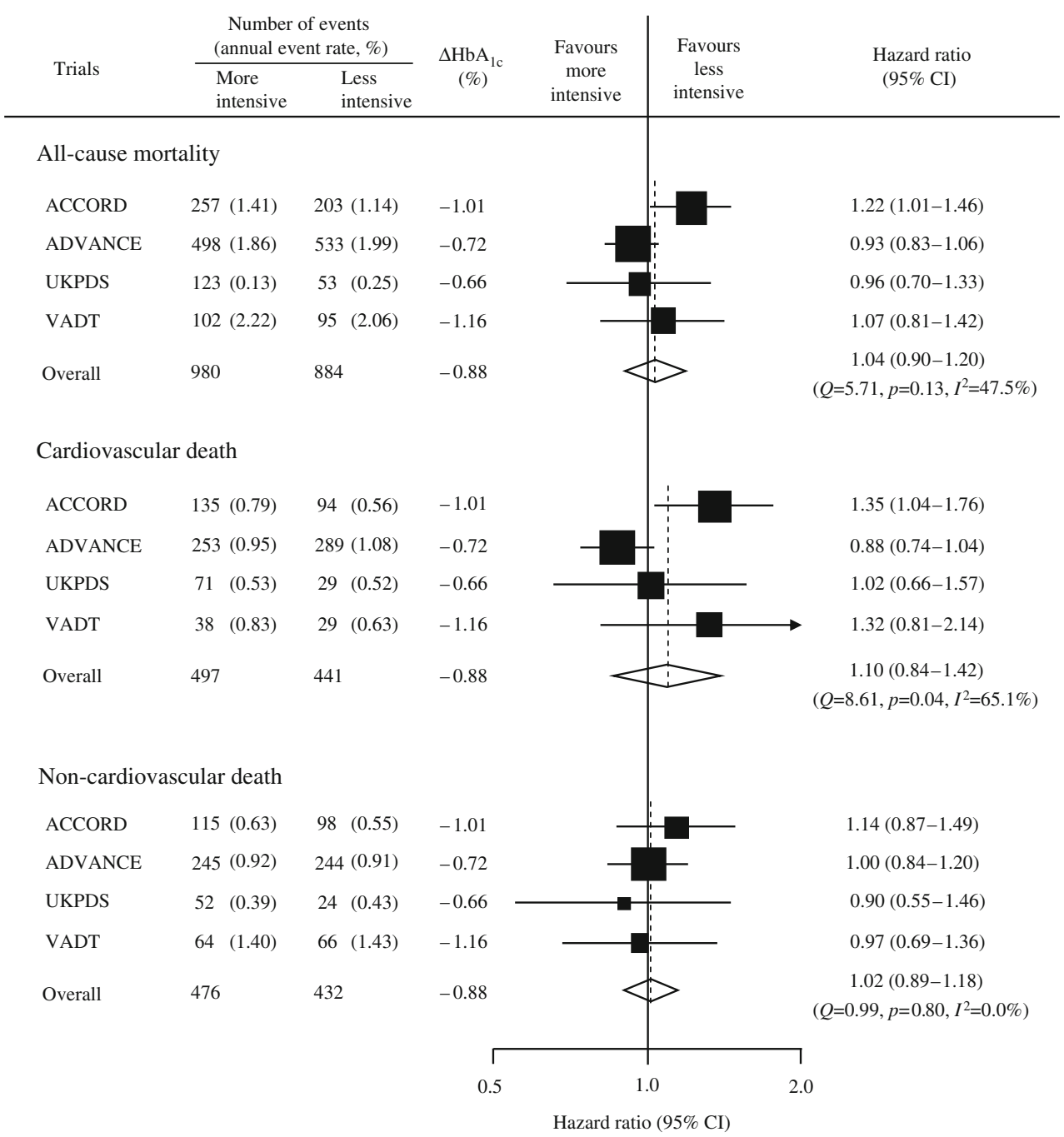


Fig. 3 Effects of more- vs less-intensive glycaemic control on major cardiovascular events for pre-specified participant subgroups. The diamond incorporates the point estimate and the $95 \% \mathrm{CI}$ of the overall effect for each outcome in each participant subgroup. The hazard ratios are given for more-intensive compared with less-intensive glucose control. ACCORD data were unavailable for the 'duration of diabetes' subgroup analyses. UKPDS follow-up truncated at 5 years from the time of randomisation

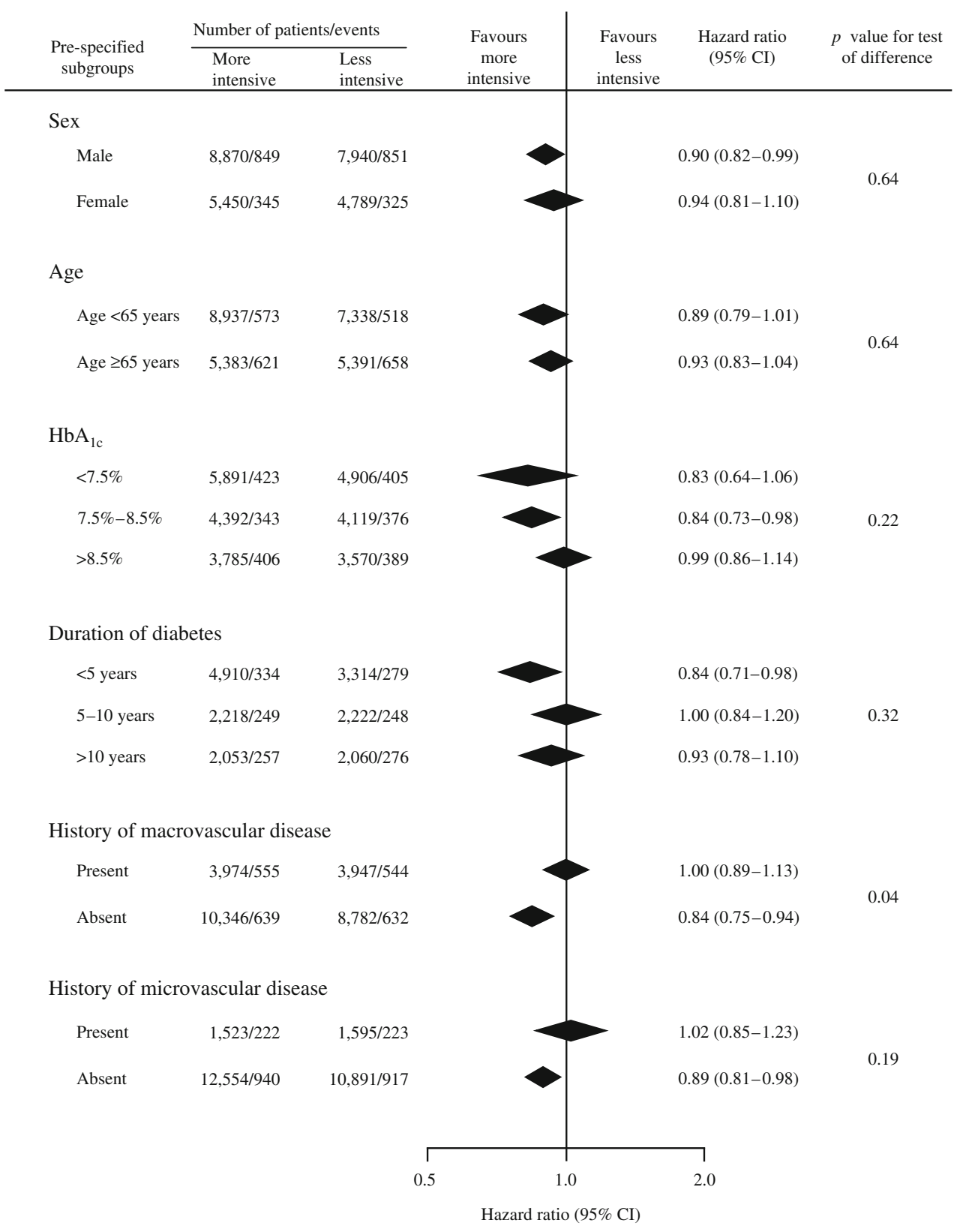

\section{Discussion}

This meta-analysis of four large, randomised controlled trials of more- vs less-intensive glycaemic control in people with type 2 diabetes demonstrates a modest reduction in major macrovascular events with greater glucose lowering. Overall, intensive glycaemic control reduced the final visit $\mathrm{HbA}_{1 \mathrm{c}}$ by a mean of 0.88 percentage points more than lessintensive glycaemic control, with an associated 9\% $(95 \%$ CI 1-16\%) RR reduction for the composite major cardiovascular outcome of cardiovascular death or non-fatal stroke or non-fatal myocardial infarction during an average follow-up of 4.4 years. For fatal/non-fatal myocardial infarction alone, the RR reduction was $15 \%(95 \%$ CI
6-24\%). The magnitude of these macrovascular risk reductions are consistent with the epidemiological relationship between $\mathrm{HbA}_{1 \mathrm{c}}$ and cardiovascular events reported from observational studies in persons with diabetes [14-17].

Meta-analysis of the other secondary endpoints showed no significant overall effect on the risk of fatal/non-fatal stroke, hospitalised or fatal congestive heart failure or allcause mortality. No significant effect was seen on cardiovascular death, although there was a $10 \%$ trend for an RR increase (HR 1.10, 95\% CI 0.84-1.42), including point estimates of 1.35 and 1.32 for the ACCORD and VADT trials, respectively. These were the two trials that achieved and maintained the greatest differences in $\mathrm{HbA}_{1 \mathrm{c}}$. The significant heterogeneity among the four studies suggests 
that the possibility of harm with more-intensive glycaemic treatment cannot be ruled out.

Exploratory subgroup analyses of the impact of moreintensive glycaemic control on the composite major cardiovascular outcome showed no significant differences with respect to sex, age, initial $\mathrm{HbA}_{1 \mathrm{c}}$, duration of known diabetes or history of microvascular disease. Although of borderline significance, there was a suggestion that participants with no history of macrovascular disease achieved benefit, whereas those with prior macrovascular disease did not.

This meta-analysis also shows that allocation to a moreintensive glycaemic control regimen is associated with a more than twofold risk of major hypoglycaemia and that many glucose-lowering medications are generally required to achieve lower glycaemic targets. Whether these factors play a role in the effect of glycaemic control on cardiovascular outcomes clearly requires further investigation. Nevertheless, in conjunction with other reported benefits of glycaemic control in patients with type 2 diabetes $[12,18]$ the results presented here suggest some cardiovascular benefit for people with diabetes. This does not preclude the possibility that the balance of risks and benefits may vary for different patient groups. Indeed, evidence of statistical heterogeneity with respect to cardiovascular death among the trials (with the highest and lowest point estimates for the HR occurring in the ACCORD and ADVANCE studies, respectively), and the benefit on the composite major cardiovascular outcome in participants without, but not in participants with, prior macrovascular disease, suggest that either patient characteristics, the approach to glucose lowering or other measured (or unmeasured) variables may affect cardiovascular risk. Avoidance of severe hypoglycaemia in the setting of an intensive glycaemic control regimen, for example, clearly requires a particular set of patient capabilities.

The chief strengths of this analysis include its focus on the key primary and secondary outcomes in the trials, the large size of the trials, the consistency of results when analysed using different approaches, and the collaboration of the original trial investigators to produce data of the highest quality. In particular, it has been possible to ensure that definitions of outcomes and exposures are directly comparable, that analytic techniques are identical across the trials, and that subgroups are defined consistently. Furthermore, sharing of the data among study groups allowed for the independent analysis and confirmation of the results.

The ability to understand the heterogeneity among trials for some outcomes, however, remains limited by the number of trials and the limited power of the subgroup analyses. However, the fact that these trials differed in several ways is apparent in Tables 1, 2, 3, 4 and 5 and some of these differences may account for the observed hetero- geneity. For example, the UKPDS was completed 10 years earlier than the other trials and studied patients with newly diagnosed vs established diabetes who were younger, lighter, more likely to be smokers, and on fewer cardioprotective drugs but at lower cardiovascular risk, and achieved a lesser contrast in the final visit $\mathrm{HbA}_{1 \mathrm{c}}$ than the other three trials. Moreover, the four trials differed with respect to their mean on-trial difference in $\mathrm{HbA}_{1 \mathrm{c}}$ (data not shown), the speed of $\mathrm{HbA}_{1 \mathrm{c}}$ lowering, the mean difference in $\mathrm{HbA}_{1 \mathrm{c}}$ reduction from baseline (Table 5), the methods by which this difference was achieved (Table 3), and the incidence rates of severe hypoglycaemia, which were also statistically heterogeneous (Table 5). They also differed with respect to the duration of exposure to the intervention, which may be an important determinant of its risks and benefits. Metaanalyses of trials using individual patient data can further explore these and other differences as possible explanations for the differential effect of intensive glucose control on myocardial infarctions vs cardiovascular death. However, these analyses were not available, as the ACCORD trial is still ongoing. Nevertheless, the exploration of the pooled data made possible by this collaboration of the four studies has generated the best estimate that is available currently of the cardiovascular benefits of more-intensive glycaemic control.

In conclusion, the recent publication of the findings from three major new trials of glucose-lowering and the UKPDS 10 year post-trial follow-up has provided important new insights into the balance of risks and benefits associated with the use of more-intensive glycaemic control in patient with type 2 diabetes. The meta-analysis presented here shows that more-intensive glycaemic control affords a modest but significant cardiovascular benefit in the short-to-medium term, although all-cause and cardiovascular mortality are not benefited. The effect on cardiovascular events is driven primarily by a $15 \%$ reduction in the risk of myocardial infarction.

Acknowledgements This study was funded by a post-doctoral Research Fellowship awarded to F. Turnbull by the National Heart Foundation of Australia.

Duality of interest H. C. Gerstein has received speaker's honoraria and consulting fees from sanofi-aventis, GlaxoSmithKline, Novo Nordisk, Roche and Lilly for topics related to the role of glucoselowering drugs for glucose control and clinical outcomes, and his institution has received grants from sanofi-aventis and GlaxoSmithKline related to clinical trials of glucose-lowering. C. Abrairia has received speaker fees from sanofi-aventis, Takeda and Novo Nordisk. J. Chalmers has received research grants from Servier, administered through the University of Sydney, as chief investigator for ADVANCE. F. Turnbull, J. Chalmers, A. Patel, B. Neal and M. Woodward have received lecture fees from Servier for speaking at scientific meetings. 


\section{References}

1. Fox C, Coady S, Sorlie P et al (2007) Increasing cardiovascular disease burden due to diabetes mellitus: the Framingham Heart Study. Circulation 115:1544-1550

2. Wild S, Roglic G, Green A, Sicree R, King H (2004) Global prevalence of diabetes: estimates for the year 2000 and projections for 2030. Diabetes Care 27:1047-1053

3. Stamler J, Vaccaro O, Neaton JD, Wentworth D (1993) Diabetes, other risk factors, and 12-yr cardiovascular mortality for men screened in the Multiple Risk Factor Intervention Trial. Diabetes Care 16:434-444

4. Huxley R, Barzi F, Woodward M (2006) Excess risk of fatal coronary heart disease associated with diabetes in men and women: meta-analysis of 37 prospective cohort studies. BMJ 332:73-78

5. Gu K, Cowie C, Harris M (1998) Mortality in adults with and without diabetes in a national cohort of the U.S. population, 1971-1993. Diabetes Care 21:1138-1145

6. Turner R, Millns H, Neil H et al (1998) Risk factors for coronary artery disease in non-insulin dependant diabetes mellitus: United Kingdom prospective diabetes study (UKPDS: 23). BMJ 316:823-828

7. The DECODE Study Group, on behalf of the European Diabetes Epidemiology Group (2003) Is the current definition of diabetes relevant to mortality risk from all causes and cardiovascular and non-cardiovascular diseases? Diabetes Care 26:688-696

8. Asia Pacific Cohort Studies Collaboration (2004) Blood glucose and risk of cardiovascular disease in the Asia Pacific region. Diabetes Care 27:2836-2842
9. The Action to Control Cardiovascular Risk in Diabetes Study Group (2008) Effects of intensive glucose lowering in type 2 diabetes. N Engl J Med 358:2545-2559

10. The ADVANCE Collaborative Group (2008) Intensive blood glucose control and vascular outcomes in patients with type 2 diabetes. N Engl J Med 358:2560-2572

11. Duckworth W, Abraira C, Moritz T et al (2009) Glucose control and vascular complications in veterans with type 2 diabetes. N Engl J Med 360:129-139

12. UK Prospective Diabetes Study (UKPDS) Group (1998) Intensive blood-glucose control with sulphonylureas or insulin compared with conventional treatment and risk of complications in patients with type 2 diabetes (UKPDS 33). Lancet 352:837-853

13. Woodward M (2005) Epidemiology, study design and data analysis, 2nd edn. Chapman and Hall, Boca Raton

14. Stratton I, Adler A, Neil H et al (2000) Association of glycaemia with macrovascular and microvascular complications of type 2 diabetes (UKPDS 35): prospective observational study. BMJ 321:405-412

15. Selvin E, Marinopoulos S, Berkenblit G et al (2004) Metaanalysis: glycosylated hemoglobin and cardiovascular disease in diabetes mellitus. Ann Intern Med 141:421-431

16. Selvin E, Coresh J, Golden S, Brancati F, Folsom A, Steffes M (2005) Glycemic control and coronary heart disease risk in persons with and without diabetes: the Atherosclerosis Risk in Communities Study. Arch Intern Med 165:1910-1916

17. Selvin E, Coresh J, Shahar E, Zhang L, Steffes M, Sharrett A (2005) Glycemia (haemoglobin A1c) and incident ischaemic stroke: the Atherosclerosis Risk in Communities (ARIC) Study. Lancet Neurol 4:821-826

18. Holman R, Paul S, Bethel M, Neil H, Matthews D (2008) Longterm follow-up after tight control of blood pressure in type 2 diabetes. N Engl J Med 359:1565-1576 